\title{
LEUCOPLASIA VERRUGOSA PROLIFERATIVA: REPORTE DE UN CASO
}

Leucoplasia verrucosa proliferativa: relatório de um caso

Fecha de Recepción: 06 de julio 2019
Proliferative verrucous leukoplakia: report of a case

Autores:
Nicolás Leonardi $^{1}$
René Luis Panico $^{1,2}$
Ruth Ferreyra $^{3}$
Ricardo Caciva $^{1,2}$

1. Cátedra Clínica Estomatológica. Escuela de Odontología. Facultad de Ciencias de la Salud. Universidad Católica de Córdoba. Argentina.

2. Cátedra de Estomatología "A".

Facultad de Odontología. Universidad Nacional de Córdoba. Argentina.

3. Cátedra Anatomía Patológica "A". Facultad de Odontología Universidad Nacional de Córdoba. Argentina.

Correspondencia:

Nicolás Leonardi

Correo electrónico: nico_leonardi@hotmail.com.ar

Conflicto de intereses:

los autores declaran no tener conflictos de interés.

Fuente de financiamiento: autofinanciado.

\section{Resumen}

La leucoplasia, es una placa blanca de la mucosa bucal, potencialmente maligna con un rango de transformación entre el 0,13\% y el $17,5 \%$. Una variante infrecuente, es la leucoplasia verrugosa proliferativa. Presenta alta probabilidad de transformarse en un carcinoma de células escamosas o un carcinoma verrugoso de Ackermann. Su etiología es incierta, pudiendo estar relacionada con el virus del papiloma humano, el virus de Epstein-Barr o incluso infecciones por cándida, pero aún no ha sido posible establecer una correlación definitiva. Se presenta el caso clínico de una paciente de género femenino de 68 años de edad derivada al Servicio de Estomatología de la Facultad de Odontología, Universidad Nacional de Córdoba donde se realizaron los estudios correspondientes para arribar al diagnóstico clínico e histopatológico compatible con leucoplasia verrugosa proliferativa.

Palabras clave: leucoplasia, leucoplasia verrugosa proliferativa, carcinoma de células escamosas, etiología, diagnóstico.

\footnotetext{
Abstract

Leukoplakia is a white plaque of the oral mucosa, potentially malignant with a transformation range between $0.13 \%$ and $17.5 \%$. An unusual variant is proliferative verrucous leukoplakia. It has a high probability of transforming into a squamous cell carcinoma or a Verrucous carcinoma of Ackerman. Its etiology is uncertain, and may be related to the human papillomavirus, the Epstein-Barr virus or even candida infections, but it has not yet been possible to establish a definitive correlation. The study presents the clinical case of a 68-year-old female patient referred to the Stomatology Service of the Dental School, National University of
} 
Córdoba. There, the corresponding studies were conducted to arrive at the clinical and histopathological diagnosis compatible with proliferative verrucous leukoplakia.

Key words: leukoplakia, proliferative verrucous leukoplakia, squamous cell carcinoma, etiology, diagnosis.

\section{Resumo}

A leucoplasia é uma placa branca da mucosa oral, potencialmente maligna, com um intervalo de transformação entre $0,13 \%$ e $17,5 \%$. Uma variante incomum é a leucoplasia verrucosa proliferativa. Tem uma alta probabilidade de se tornar um carcinoma espinocelular ou um carcinoma mais quente de Ackermann. Sua etiologia é incerta e pode estar relacionada ao vírus do papiloma humano, vírus Epstein-Barr ou até infecções por candida, mas ainda não foi possível estabelecer uma correlação definitiva. Apresenta-se o caso clínico de uma paciente de 68 anos, encaminhada ao Serviço de Estomatologia da Faculdade de Odontologia da Universidade Nacional de Córdoba, onde foram realizados os estudos correspondentes para chegar ao diagnóstico clínico e histopatológico compatível com leucoplasia verrucosa proliferativa.

Palavras-chave: leucoplasia, leucoplasia verrucosa proliferativa, carcinoma espinocelular, etiologia, diagnóstico.

\section{Introducción}

La leucoplasia verrugosa proliferativa (LVP) fue descripta por primera vez por Hansen et al. ${ }^{1}$, como un tipo específico de leucoplasia oral que presenta características clínicas distintivas. Presentan un comportamiento biológico agresivo con tendencia a la multifocalidad, alta tasa de recidiva después del tratamiento, y de transformación maligna, pudiendo progresar ya sea a un Carcinoma Verrugoso o a un Carcinoma Espino Celular.

Hansen et al. ${ }^{1}$, describieron la LVP como una enfermedad de etiología poco clara, pero típicamente asociada con el consumo de tabaco. Sin embargo, el papel del tabaco en las lesiones de LVP es descono- cido ya que estas lesiones se observan en fumadores y no fumadores.

Inicialmente, la LVP se observa como una placa blanca que no se desprende al raspado, de crecimiento lento, que con el tiempo se vuelve verrugosa y exofítica, pudiendo tener áreas eritematosas y llegando a comprometer diversas partes de la cavidad bucal, como el paladar duro y blando, mucosa alveolar, lengua, piso de boca y encía ${ }^{2-4}$.

Las mujeres se ven más afectadas que los hombres, sobre todo después de los 60 años $^{3}$. No existe una predilección racial ${ }^{5}$. A pesar de los diversos intentos por asociarla a un factor determinado como el tabaco, virus Papiloma Humano (VPH), virus de Epstein-Barr, o con infecciones por cándida, aún no se ha podido detectar una relación válida con ninguno de ellos ${ }^{6}$.

Dentro de la clasificación de la OMS, la LVP es también considerada dentro de los desórdenes potencialmente malignos, que requiere un reconocimiento temprano, para tratar de evitar su transformación, siendo que la lesión es resistente a todo tratamiento ${ }^{6}$.

Desde 1985 hasta la fecha, la LVP se ha mantenido como una entidad nosológica propia y compleja. Su etiología aún sigue siendo desconocida ${ }^{7-11}$. Es de difícil diagnóstico, tanto clínico como histopatológico, ya que puede confundirse con una leucoplasia homogénea, leucoplasia verrugosa, eritroleucoplasia, carcinoma verrugoso, liquen plano, entre otras ${ }^{8-11}$. No existe un consenso sobre criterios diagnóstico o protocolos de tratamiento aceptados, lo que hace difícil establecer comparaciones entre los diversos estudios publicados en la literatura ${ }^{8}$.

\section{Caso clínico}

Paciente de sexo femenino, 68 años de edad, nacionalidad argentina, derivada al servicio de Estomatología "A" de la Facultad de Odontología de la Universidad Nacional de Córdoba. Respecto a los antecedentes personales patológicos los datos de relevancia son hipotiroidismo medicada con levotiroxina $75 \mathrm{mg}$, hipertensión arterial medicada con losartan potásico $50 \mathrm{mg}$, mal de Chagas diagnosticada 


\section{9}

CASO CLÍNICO
Revista de la Facultad de Odontología

Leucoplasia verrugosa proliferativa: Reporte de un caso

\section{REFO}

VOL. XIII | No 1| AÑO 2020

ISSN 1668-7280

ISSN-E 2683-7986 hace 40 años, medicada con acalix cd 180mg y aldator $25 \mathrm{mg}$. Actualmente es fumadora pasiva, pero ha fumado durante 40 años, 73000 cigarrillos rubios con filtro aproximadamente. No relata consumo de alcohol, drogas y mate.

Al examen clínico se pudo observar la presencia de una mancha blanca con áreas queratoticas y verrugosas que se extienden afectando los tres tercios de mucosa yugal derecha, áspera al tacto lingual, con una zona roja eritroplásica que alcanzaba el reborde alveolar y fondo de surco lado derecho. Sus límites son nítidos y bordes irregulares, 1 año de evolución, sin sintomatología, ni tratamiento previo (Figura 1).
Se solicitaron estudios complementarios de diagnóstico, tales como análisis de rutina arrojando valores normales y ortopantomografía sin datos de relevancia. Se planteó posible diagnóstico presuntivo y sus respectivas alternativas diagnósticas.

Tras la evaluación clínica y los exámenes complementarios, se realizó una biopsia incisional del sitio más representativo de la lesión, incluyendo áreas blancas y la zona eritroplásica (Figura 2). Se obtuvo un fragmento de tejido de mucosa yugal derecha, se fijó en formol al $10 \%$ y se realizó el análisis de la misma en el servicio de Anatomía Patológica de la Facultad de Odontología, Universidad Nacional de Córdoba. Se informó que los cortes histológicos mostraban un epitelio pavimentoso estratificado

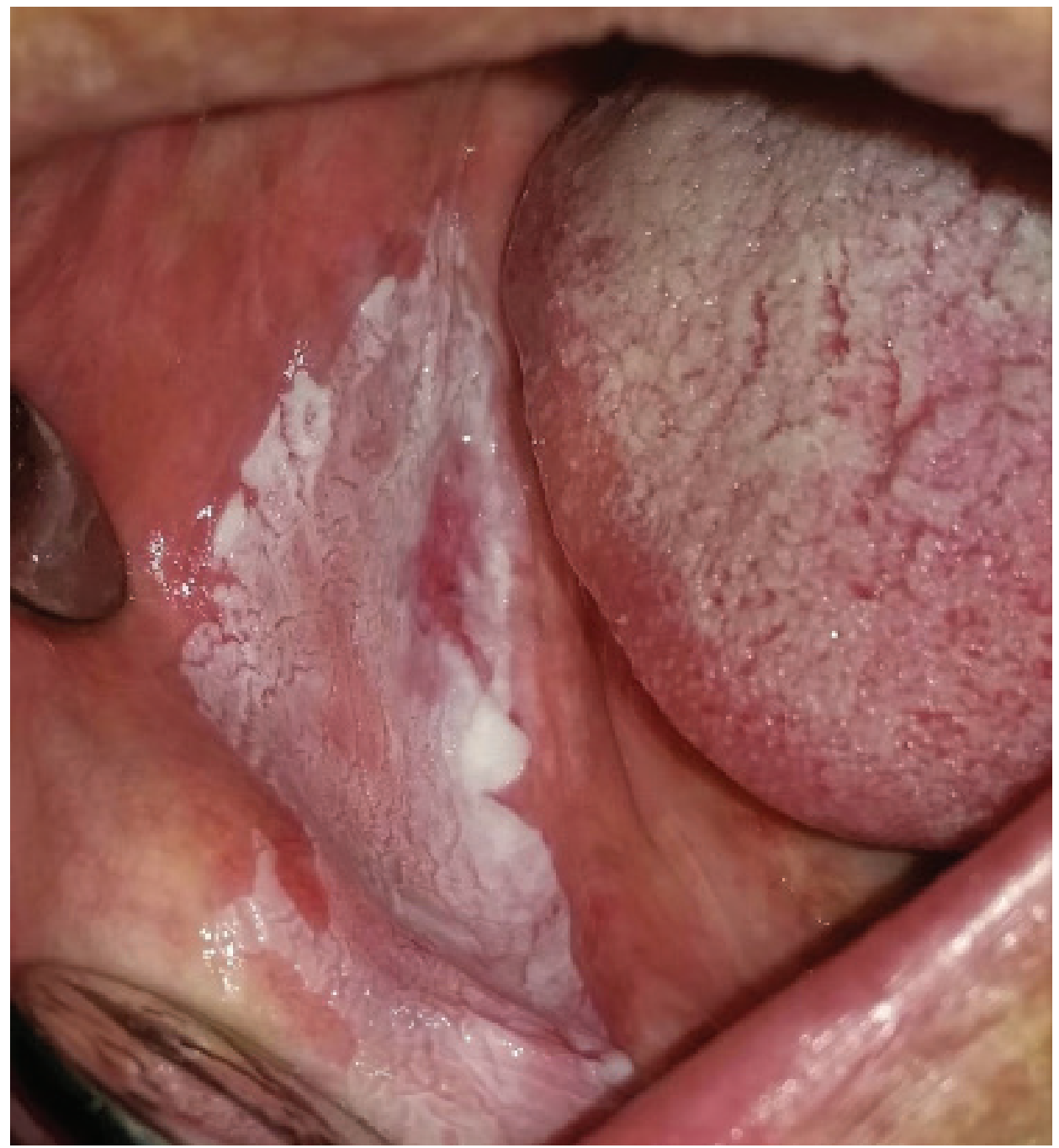

Figura 1. Examen Intrabucal. 
hiperortoqueratinizado con sectores hiperparaqueratinizados con formaciones cupuliformes, algunas imágenes "tipo coilociticas", granulosis y acantosis (Figura 3). Presentaba una zona de displasia epitelial moderada con crestas interpapilares en forma de gotas. Por debajo infiltrado linfocitario yuxtaepitelial, tejido fibrocolágeno con trayectos vasculares dilatados, tejido celular subcutáneo y fibras musculares estriadas, confirmando un cuadro compatible con leucoplasia verrugosa proliferativa (Figura 4).

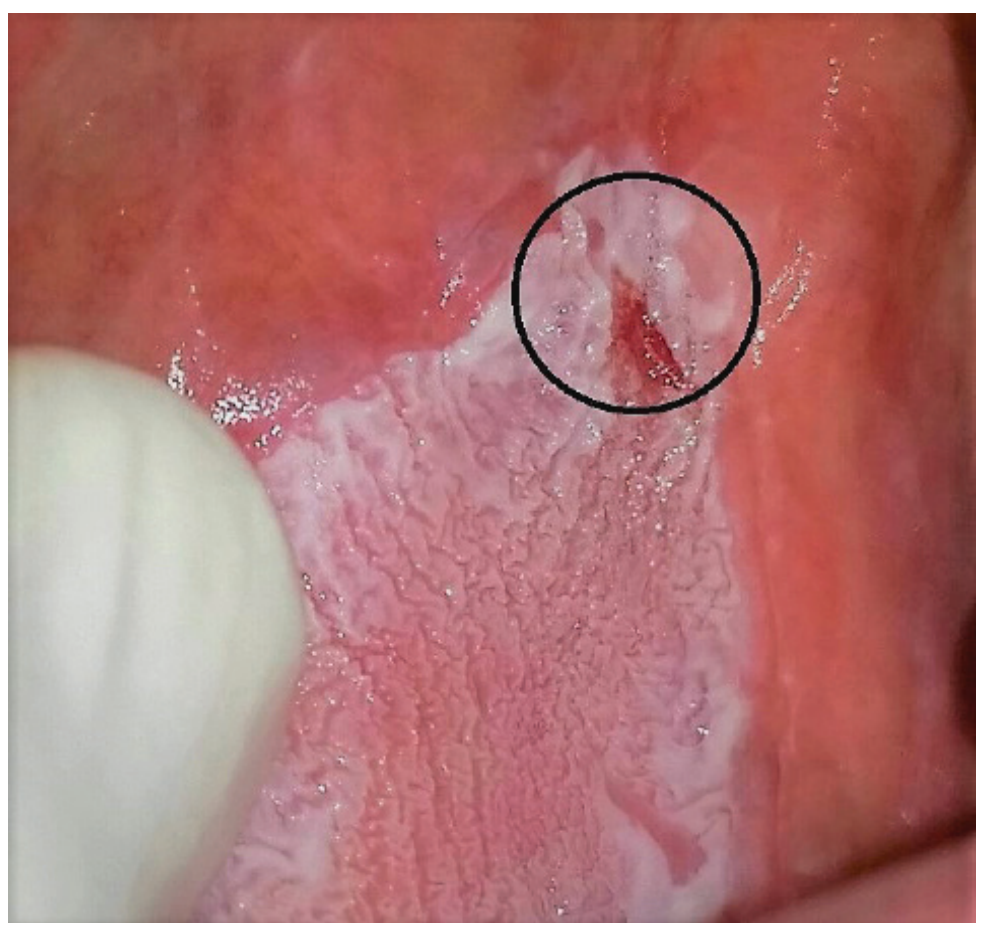

Figura 2. Zona de biopsia incisional..

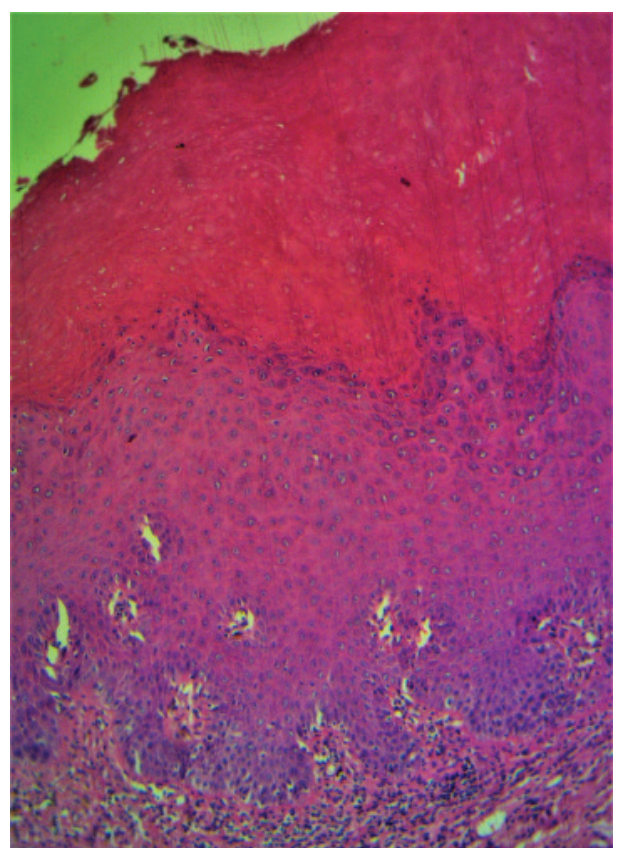

Figura 3. Leucoplasia verrugosa proliferativa. HE 200X. Presenta hiperortoqueratosis marcada, granulosis, red de crestas interp pilares en forma de gotas. El corion presenta infiltrado linfocitarios y melanófagos perivasculares.

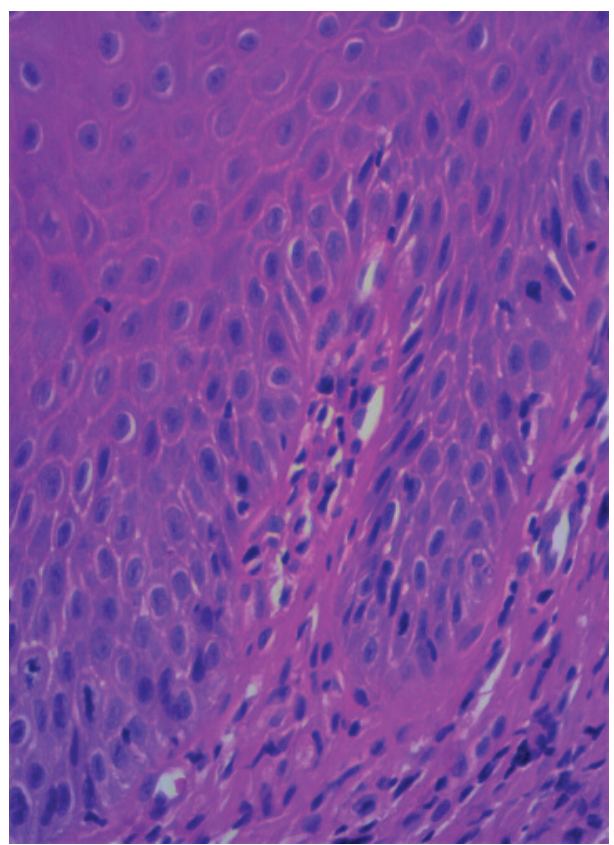

Figura 4. Leucoplasia verrugosa proliferativa. HE 400X. Cambios displásicos, con hiperplasia de estratos inferiores y células con pleomorfismo celular. En el corion se observa el infiltrado linfocitario yuxtaepitelial. 
Luego que se arribó al diagnóstico de certeza se realizó la correspondiente derivación a cirujano especialista en cabeza y cuello para la escisión completa de la lesión. La evolución del caso clínico presentado fue favorable, con exhaustivos controles periódicos.

\section{Discusión}

La importancia del caso clínico presentado radica en la relevancia de realizar una oportuna derivación a los profesionales especializados por parte del odontólogo general y así generar un correcto diagnóstico y su posterior tratamiento. Estos pacientes deben estar monitoreados periódicamente debido al gran riesgo de transformación maligna de la leucoplasia verrugosa proliferativa en carcinoma bucal de células escamosas. Consideramos que es fundamental un diagnóstico precoz de estas lesiones ya que la demora en el mismo es la principal dificultad en el tratamiento, siendo éste investigado en la actualidad como la o las causas idiopáticas que podrían llegar a estar asociadas a esta entidad.

La LVP es un desorden epitelial que lleva hacia el carcinoma bucal. No se ha logrado un tratamiento eficaz, tan sólo detención durante períodos de tiempo. La LVP presenta, como mayor dificultad del tratamiento, el diagnóstico tardío ya que la presentación clínica e histopatológica se inicia como una lesión sin alteraciones celulares las cuales van apareciendo en la evolución de la lesión. El riesgo de transformación maligna de la LVP varía entre $70 \%$ y $87 \%$ mientras que en la leucoplasia convencional, el rango de transformación maligna es de sólo 5\%3,6,7,9.

Para el diagnóstico del caso presentado, se siguieron las pautas establecidas por Cerero-Lapiedra et al. ${ }^{4}$, quienes propusieron para el diagnóstico definitivo de LVP, 5 criterios mayores y 4 menores y combinaciones entre ellos. Los criterios mayores son: A. Lesión leucoplásica en más de dos sitios de la cavidad bucal, más frecuentemente en la encía, proceso alveolar y paladar; B. Existencia de área verrugosa; C. Que las lesiones se hayan expandido o engrosado durante el desarrollo de la enfermedad; D. Que haya habido recidivas en áreas previamente tratadas; E. Puede ser desde la forma de hiperqueratosis epitelial simple a hiperplasia verrugosa, carcinoma verrugoso o carcinoma escamoso ya sea in situ o infiltrante. Los criterios menores son: A. Lesión bucal leucoplásica que ocupe por lo menos 3 centímetros si se suman todas las áreas afectadas; B. Paciente del sexo femenino; $C$. Paciente no fumador; D. Enfermedad de más de cinco años de evolución. Para realizar el diagnóstico de LVP, se sugiere una de las combinaciones: 1 . Tres criterios mayores (debe considerar el E entre ellos); 2. Dos criterios mayores (debe estar el E entre ellos) más dos criterios menores.

Aguirre-Urizar ${ }^{12}$, basándoseen las características clínicas de este desorden potencialmente maligno, propone cambiar su nombre por el de "leucoplasia proliferativa multifocal”, resaltando la importancia que tiene desde los comienzos el hecho de que las lesiones son proliferativas y múltiples.

\section{Referencias Bibliográficas}

1. Hansen LS, Olson JA, Silverman S Jr. Proliferative verrucous leukoplakia. A long term study of thirty patients. Oral Surg Oral Med Oral Pathol. 1985;60(3):285-98. doi: 10.1016/0030-4220(85)90313-5

2. Bagan J, Scully C, Jimenez Y, Martorell, M. Proliferative verrucous leukoplakia: A concise update. Oral Dis. 2010;16(4):328-32. doi: 10.1111/j.16010825.2009.01632.x

3. Cabay R J, Morton TH Jr, Epstein JB. Proliferative verrucous leukoplakia and its progression to oral carcinoma: a review of the literature. J Oral Pathol Med. 2007; 36(5):255-61. doi: 10.1111/j.1600-0714.2007.00506.x

4. Cerero-Lapiedra R, Baladé-Martínez D, Moreno-López LA, Esparza-Gómez G, Bagán JV. Proliferative verrucous leukoplakia: a proposal for diagnostic criteria. Med Oral Patol Oral Cir Bucal. 2010;15(6):839-45. doi:10.4317/ medoral.15.e839

5. Ghazali N, Bakri MM, Zain RB. Aggressive, multifocal oral verrucous leukoplakia: Proliferative verrucous leukoplakia or not? J Oral Pathol Med. 2003;32(7):383-92. doi: 10.1034/j.1600-0714.2003.00180.x

6. Warnakulasuriya S, Johnson NW, van der Waal I. Nomenclature and classification of potentially malignant disorders of the oral mucosa. J Oral Pathol Med. 2007;36(10):575-80. doi: 10.1111/j.16000714.2007.00582.x

7. van del Waal I, Reichart PA. Oral proliferative leukoplakia revisited. Oral Oncol. 2008;44(8):719-21. doi: 
10.1016/j.oraloncology.2007.09.010

8. Bagan JV, Jiménez Y, Murillo J, Poveda R, Díaz JM, Gavaldá C, et al. Epstein-Barr virus in oral proliferative verrucous leukoplakia and squamous cell carcinoma: A preliminary study. Med Oral Patol Oral Cir Bucal. 2008;13(2):110-3. PMID: 18223526

9. Navarro CM, Sposto MR, Sgavioli-Massucato EM, Onofre MA. Transformation of proliferative verrucous leukoplakia to oral carcinoma: a ten years follow-up. Med Oral. 2004;9(3):229-33. PMID: 15122125

10. Gouvêa AF, Vargas PA, Coletta RD, Jorge J, Lopes MA. Clinicopathological features and immunohistochemical expression of p53, Ki-67, Mcm-2 and Mcm-5 in proliferative verrucous leukoplakia. J Oral Pathol Med. 2010;39(6):447-52. doi: 10.1111/j.16000714.2010.00889.x

11. Liu W, Shen XM, Liu Y, Li J, Zhou ZT, Wang LZ. Malignant transformation of oral verrucous leukoplakia: a clinicopathologic study of 53 cases. J Oral Pathol Med. 2011;40(4):312-316. doi: 10.1111/j.16000714.2011.01016.x

12. Aguirre-Urizar JM. Proliferative multifocal leukoplakia better name that proliferative verrucous leukoplakia. World J Surg Oncol. 10;9:122,2011. doi: 10.1186/14777819-9-122 